\title{
Are corticosteroids useful in all degrees of severity and rapid recovery of Bell's palsy?
}

\author{
Margarida Ferreira ${ }^{\mathrm{a}, \mathrm{b}}$, Machado J. Firmino ${ }^{\mathrm{c}}$, Elisa A. Marques ${ }^{\mathrm{d}, \mathrm{e}}$, Paula C. Santos ${ }^{\mathrm{a}, \mathrm{f}}$ and José A. Duarte ${ }^{\mathrm{a}}$ \\ ${ }^{a}$ Research Centre in Physical Activity, Health and Leisure, Faculty of Sport, University of Porto, Porto, Portugal; ${ }^{b}$ Department of Physiotherapy, \\ Paredes and Vila Nova De Famalicão, North Polytechnic Institute of Health, CESPU, Portugal; 'Department of Public Health - Occidental \\ Oporto, Portugal; ${ }^{d}$ Research Center in Sports Sciences, Health and Human Development (CIDESD), University Institute of Maia (ISMAI),

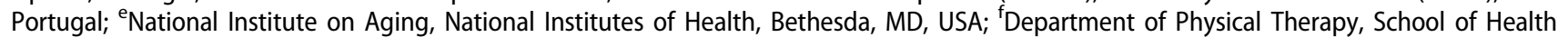 \\ Technology of Porto, Polytechnic Institute of Porto, Vila Nova De Gaia, Portugal
}

\begin{abstract}
Conclusions The results provide preliminary evidence that corticosteroids were not effective in all grades of dysfunction and for achieving a rapid remission in the early phase of BP, highlighting the need to define standard and rigorous criteria to prescribe corticosteroids in these patients. Objectives The main aim of this study was to investigate whether the use of corticosteroids better associated than paralleled with neuromuscular training $(C+F N T)$ is more effective than facial neuromuscular training (FNT) applied alone, in terms of recovery degree and facial symmetry during the early phase of Bell's palsy (BP). Patients and methods A prospective single-blinded study involved 73 patients: the $C+$ FNT group ( $n=42$; median age $=37.5$ years) and FNT group $(n=31$; median age $=49.0$ years). Patients were assessed before and 6 weeks after treatment by House-Brackmann (HB-FGS) and Sunnybrook Facial Grading System (SB-FGS). Results Recovery degree and facial symmetry improved significantly in both groups $(p<0.001)$, without differences between groups $(p>0.05)$. However, the $C+$ FNT group displayed better outcomes for cheek $(p=0.004)$ and mouth $(p=0.022)$ resting symmetry at SB-FGS, instead of compared to the FNT group. The corticosteroids had no significant effect on all recovery degrees $(p=0.992)$ and rapid remission $(p=0.952)$. Multiple linear regression analysis showed that the type of intervention was not a significant predictor for recovery degree $(p=0.917)$.
\end{abstract}

\section{KEYWORDS \\ Facial palsy; medical \\ therapy; muscular training; Sunnybrook Facial Grading System; House-Brackmann Facial Grading System}

\section{Introduction}

Bell's palsy (BP) is defined as an acute unilateral paralysis or paresis of the facial nerve without any associated disorders [1]. Incomplete recovery from facial nerve damage has significant social and psychological consequences [2]. Although the prognosis of $\mathrm{BP}$ is generally good, with a high rate of recovery, $29 \%$ of patients were reported as having sequels [1]. Moreover, patients with facial paralyses and absence of recovery within 3 weeks may experience poor outcomes [1].

Pathologically HSV-1 (re)activation results in an inflammation of the seventh facial nerve, which leads to compression within the bony canal fallopian and nerve demyelination [3]. Based on this, the American Academy of Neurology [4] established that powerful anti-inflammatory agents, such as oral corticosteroids, are effective in improving facial function outcomes in BP. Furthermore, Sullivan et al. [5] reported a significant improvement of facial nerve function in patients treated with prednisolone within $72 \mathrm{~h}$ of onset. On the other hand, systematic reviews have concluded that the recommendation for optimal physical therapy of BP is difficult due to the limited number and low quality of trials and the heterogeneity of results [6-8].

The recent Canadian Guidelines recommend the use of corticosteroids for all patients with BP [9]. Moreover, Indian
Guidelines include physical therapy and corticosteroids for targeting the speed recovery [10]. Thus, there are no data regarding the effects of corticosteroids treatment vs facial neuromuscular training only, in the early phase of BP.

In this study, the main goal was to investigate whether the use of corticosteroids with neuromuscular training is more effective than neuromuscular training alone, in terms of recovery degree and facial symmetry during the early phase of BP. Four main hypotheses were tested: (1) corticosteroids treatment influences positively the recovery degree and facial symmetry; (2) patients with a high degree of severity have more benefits with the corticosteroids; (3) recovery is faster with the corticosteroids; and (4) predictors, such as age, sex, baseline HB-FGS, and intervention type, influence the degree of recovery.

\section{Methods \\ Subjects and experimental design}

The study was a prospective single-blinded study performed in patients with peripheral facial paralysis, screened from January 2009 to May 2013. The first clinical visit included examinations by a clinical physician, an otolaryngologist, and 
a physiatrist, according to the routine protocol of hospitalPortugal. In the Emergency department, all outpatients were referred to Physical Medicine and Rehabilitation by the clinical physician. The protocol was approved by the hospital ethics committee, and written informed consent was obtained from all studied patients. Baseline sample was 123 patients with peripheral facial paralysis, diagnosed by a physiatrist. The eligibility criteria for this study included: patients with unilateral $\mathrm{BP}$, both gender, aged 18 years and older, onset of oral corticosteroids treatment within $72 \mathrm{~h}$ after BP onset, and facial neuromuscular training treatment within 10 days after BP onset. Patients were excluded if they had recurrent facial nerve palsy, a central nervous system disease, traumatic facial nerve injury, metabolic, infective, cancer, psychiatric condition, neuropathy or neuromuscular disease, antiviral treatment therapy, corticosteroids treatment for others diseases, and the inability to speak or read Portuguese language.

BP was diagnosed when no other cause was identified through medical history and physical examination. In case of a diagnostic disagreement, radiology and laboratory testing were performed. A questionnaire was applied to all patients, which included demographic data, side of palsy, symptoms, first consultation, and medical and personal history. After the diagnosis of BP, 73 patients were divided into two treatment groups: the Corticosteroids plus Facial Neuromuscular Training $(\mathrm{C}+\mathrm{FNT})$ group or the Facial Neuromuscular Training (FNT) group. The prescription criteria for corticosteroids administration were unknown and without any apparent uniformity among the physicians, showing that all patients take corticosteroids independently of the level of severity of PB. Blinding to treatment allocation of participants and physical therapists was not possible due to the nature of the interventions. However, clinical physicians and investigators were blind to the allocated intervention.

\section{Intervention}

The C + FNT group includes patients taking oral prednisolone, within $72 \mathrm{~h}$ of BP onset, at a dose of $1 \mathrm{mg} / \mathrm{kg}$ per day (maximum $60 \mathrm{mg} /$ day) for 5 days, then tapered over 5 days $(10 \mathrm{mg} /$ day). FNT was started within 10 days in all patients, no difference was shown for the timing of the start of the intervention between groups $(4.98 \pm 2.38$ and $5.29 \pm 2.3$, $p=0.775$ for $\mathrm{C}+\mathrm{FNT}$ and FNT, respectively). Both groups were treated with FNT individually by five trained physical therapists in accordance to the facial neuromuscular training principles [11]. Each patient was treated in the outpatient hospital, 5 days a week, 20 min a day, for 6 weeks or until complete recovery. In the case of complete recovery, FNT was no longer performed, although assessment continued until the sixth week.

A standard program of FNT included different muscle groups supplied by each branch of the facial nerve in both sides of the face by using a mirror to promote symmetry and feedback. The FNT was based on principles of strength training, overload and specificity [12]. Table 1 shows the detailed description of each training phase.

\section{Outcome measures}

Assessment of patients was performed using two the HouseBrackmann facial grading system (HB-FGS) and Sunnybrook facial grading system (SB-FGS) [13,14]. The HB-FGS consists of six grades, where grade I represents normal facial function in all areas, and grade VI represents total paralysis. This scale was assessing symmetry, synkinesis, stiffness, and mobility of the face. Complete recovery was defined as the achievement of HB-FGS grade I [13]. Facial symmetry was measured using the 13-item SB-FGS. A composite facial symmetry score is calculated as $[(4 \times$ symmetry of voluntary movement score $)$ - $(5 \times$ resting asymmetry score $)+(1 \times$ synkinesis score)], with 100 points representing normal facial symmetry [14]. The score for three sections are combined into a composite facial symmetry score by subtracting the resting asymmetry and synkinesis. In the present study, the synkinesis component could not be established due to the absence of synkinesis in the early phase of BP (score 0/none synkinesis). However, the absence of this component does not change the sum of final score. Outcome measurements were assessed at baseline and after 6-weeks of follow-up period.

\section{Data analysis}

Categorical variables are presented as absolute and relative frequencies, while continuous variables are presented as absolute values, using means and standard deviations, or medians and interquartile ranges for variables with skewed distributions. Differences between two interventions (i.e. C + FNT vs FNT) in baseline characteristics were tested using $\chi^{2}$ test and Mann-Whitney U-test, as appropriate. HB-FGS and SB-FGS scores were analyzed by Kruskall-Wallis test to compare differences within- and between-groups. The median variation of recovery degree, i.e. $\Delta$ HB-FGS and $\Delta$ SB-FGS (final valueinitial value), was tested among groups using the MannWhitney test. $\chi^{2}$ test analyzed the differences in proportions of patients between groups (C+FNT vs FNT groups) who experienced full recovery (HB-FGS grade I) as well as after stratifying patients according to their baseline HB-FGS grades (slight-to-moderate severity grade II, III, IV; severe severity grade $\mathrm{V}, \mathrm{VI}$ ). The remission time was assessed every week by the number of days to reach HB-FGS grade I, which was analyzed through hazard curves using Log-Rank Test for differences among groups. Multiple linear regression was performed to test an association between predictor intervention group and outcome variable of the $\triangle \mathrm{HB}-\mathrm{FGS}$, adjusting for confounding variables (age, sex, and severity baseline of HBFGS). $p$-values less than 0.05 were considered as significant. Analyses were performed using SPSS, version 22.0.

\section{Results}

All demographic and baseline clinical measures were similar in both groups $(p>0.05)$ (Table 2). 
Table 1. Facial neuromuscular training components of intervention.

\begin{tabular}{|c|c|}
\hline Phase 1 Information & $\begin{array}{l}\text { All patients were educated on facial muscle physiology, facial functions and changes induced by } \\
\text { paralysis }\end{array}$ \\
\hline \multirow[t]{4}{*}{ Content of the standardized education } & $\begin{array}{l}\text { Postural correction information was given for to increase awareness of the head position and its effect } \\
\text { on facial tone }\end{array}$ \\
\hline & $\begin{array}{l}\text { Relaxation training was taught to increase the participant's awareness of the hyperactivity of the } \\
\text { unaffected face by breathing and relaxation exercises }\end{array}$ \\
\hline & $\begin{array}{l}\text { Tissue mobilization was taught to improve circulation and sensory stimulation by combination of } \\
\text { 'gliding' and 'kneading', in both side of the face and neck during 5-10 min }\end{array}$ \\
\hline & $\begin{array}{l}\text { Strategies were instructed to avoid mass facial movements (excessive chewing on the affected } \\
\text { side), eye protection (wear glasses, an eye shield, and ointment), and oral hygiene }\end{array}$ \\
\hline
\end{tabular}

Phase 2

Training components and techniques intervention Standardized training components

Techniques intervention
All patients were treated individually by a trained physical therapist

Homework

\begin{abstract}
The basis exercises consisted of seven facial muscles/motion (Frontalis/wrinkling; Corrugator supercili/ wrinkles of forehead; Orbicularis oculi/closure of eyes; Procerus/snarl; Orbicularis oris/whistling; Risorius/smile; Zigomaticus/laugh) with variations of speed, amplitude, force, repetitions (from 5-20), series (from 1-2) per each facial muscle, selective and co-ordination motion and other muscles innervated by the facial nerve. Patients were also taught expression exercises (e.g. to open the eyes wide/surprise, lift the upper lip/disgust, tighten the lips/anger, lips pucker/kiss or laugh/ happy) and facial function (eat, drink, speak)

Neuromuscular facial training had two different interventions according to the House-Brackmann-Facial Grading System assessment. Patients who had severe facial asymmetry at rest or flaccid facial regions, and who were unable to initiate movement on the affected side were given instructions on small and controlled movement by passive and active assistive exercises. On the other hand, patients who had mild-to-moderate facial asymmetry at rest and were able to initiate at least slight movement in any or all regions of the face were given instructions in the isolated movement and controlled by active assistive and resistive exercises

In addition, when the patients performed correct movements and symmetry of facial expression, they were instructed to practice a home-based exercise program, 2 times a day, for no more than 10 min per day. Adherence to the home exercise was formally registered daily in a standard document by patients and it was weekly controlled by physical therapists.
\end{abstract}

Table 2. Demographic and clinical data in both groups.

\begin{tabular}{lccc}
\hline & $\begin{array}{c}\text { C }+\begin{array}{c}\text { FNT group } \\
(n=42)\end{array} \\
\text { Sex, } n \text { (\%) }\end{array}$ & $\begin{array}{c}\text { FNT group } \\
(n=31)\end{array}$ & $p$-value \\
$\quad$ Male & $19(45.24)$ & $16(51.61)$ & 0.593 \\
$\quad$ Female & $23(54.76)$ & $15(48.39)$ & \\
Face side, $n$ (\%) & & & \\
$\quad$ Right & $20(47.62)$ & $13(41.94)$ & 0.632 \\
$\quad$ Left & $22(52.38)$ & $18(58.06)$ & \\
Age (years), Median (IQR) & $37.5(26)$ & $49.0(33)$ & 0.053 \\
Baseline HB-FGS, $n$ (\%) & & & 0.944 \\
$\quad$ Grade II & $5(11.90)$ & $3(9.68)$ & \\
$\quad$ Grade III & $12(28.57)$ & $9(29.03)$ & \\
$\quad$ Grade IV & $5(11.90)$ & $4(12.90)$ & \\
$\quad$ Grade V & $4(9.52)$ & $5(16.13)$ & \\
$\quad$ Grade VI & $16(38.10)$ & $10(32.26)$ & \\
\hline
\end{tabular}

IQR: interquartile range; HB-FGS: House-Brackmann facial grading system; SBFGS: Sunnybrook facial grading system; C + FNT: corticosteroids plus facial neuromuscular training group; FNT: facial neuromuscular training group.

According to the HB-FGS, the median paresis severity at baseline was grade 4 for both groups. On the SB-FGS, median score at baseline was 26 points in the $\mathrm{C}+\mathrm{FNT}$ group and 30 points in the FNT group (Table 3). As shown in Table 3, both groups significantly improved all components of facial symmetry (within-group $p<0.05$ ). However, the $\mathrm{C}+\mathrm{FNT}$ group displayed better outcomes for cheek $(p=0.004)$ and mouth $(p=0.022)$ resting symmetry compared to FNT group (Table 3 ). Composite facial symmetry score improved 74 points in the $\mathrm{C}+\mathrm{FNT}$ group compared to 70 points in the FNT group.

After 6 weeks, the clinical data indicate that both groups showed significant improvements of recovery degree $(\Delta \mathrm{SB}$ FGS), without significant differences between groups $(p>0.05)$ (Table 4$)$. Both groups increased $\sim 2^{\circ}$ HB-FGS, while $\triangle$ SB-FGS median showed 54.50 points to the $\mathrm{C}+\mathrm{FNT}$ and 47.0 to the FNT group.
No significant effect of both interventions was found between groups. Similarly, no differences were found after stratifying patients according to their baseline HB-FGS grades (II-IV and V-VI). As shown in Table 5, at the end of the follow-up period, 27 (64.29\%) patients in the C+FNT group and $20(64.52 \%)$ in the FNT group experienced full recovery $(p=0.992)$.

There were no differences in remission time (reaching HB-FGS grade I) between groups $(p=0.952)$ (Figure 1$)$. The average remission time was 17.86 days $(95 \%$ $\mathrm{CI}=15.68-20.03)$ in the $\mathrm{C}+\mathrm{FNT}$ group and 18.43 days $(95 \% \mathrm{CI}=15.23-21.63)$ in the FNT group.

Considering the recovery degree $(\Delta \mathrm{HB}-\mathrm{FGS})$ in all patients, a multiple linear regression model was performed adjusted for variables such as age, sex, baseline HB-FGS, and intervention groups. Age, sex, and baseline HBFGS were significant confounding variables $(p<0.004$, $p<0.003$, and $p<0.001$, respectively). The type of intervention was not a significant predictor of recovery degree $(p=0.917)$.

After adjusting for age, sex, and baseline HB-FGS, the type of intervention was not a significant predictor of recovery degree $(\Delta \mathrm{HB}-\mathrm{FGS} ; p=0.917)$ (Table 6$)$.

\section{Discussion}

The results of the present study show that oral administration of corticosteroids does not bring advantageous effects in terms of recovery degree and facial symmetry in the early phase of BP. Moreover, we found some heterogeneity in corticosteroids prescription among physicians, since they were 
Table 3. Baseline and after intervention expressed by HB-FGS and SB-FGS components, in both groups.

\begin{tabular}{|c|c|c|c|c|c|c|c|}
\hline \multirow[b]{2}{*}{ Scales } & \multicolumn{3}{|c|}{$\mathrm{C}+\mathrm{FNT}$ group } & \multicolumn{3}{|c|}{ FNT group } & \multirow[b]{2}{*}{$p$-value +} \\
\hline & Baseline & After intervention & $p$-value* & Baseline & After intervention & $p$-value* & \\
\hline HB-FGS (I to VI grade) & $4.00(3.0-6.0)$ & $1.00(1.0-3.0)$ & $<0.001$ & $4.00(3.0-6.0)$ & $1.00(1.0-2.0)$ & $<0.001$ & 0.665 \\
\hline Composite facial symmetry score $(0-100)$ & $26.00(5.0-60.8)$ & $100.00(83.8-100)$ & $<0.001$ & $30.00(10.0-60.0)$ & $100.00(79.00-100)$ & $<0.001$ & 0.794 \\
\hline Eye $(0-1)$ & $1.00(0.0-1.0)$ & $0.00(0.0-0.0)$ & $<0.001$ & $1.00(0.0-1.0)$ & $0.00(0.0-1.0)$ & 0.001 & 1.000 \\
\hline Cheek (0-2) & $0.00(0.0-1.0)$ & $0.00(0.0-0.0)$ & $<0.001$ & $1.00(0.0-1.0)$ & $0.00(0.0-0.0)$ & 0.001 & 0.004 \\
\hline Mouth $(0-1)$ & $0.00(0.0-1.0)$ & $0.00(0.0-0.0)$ & $<0.001$ & $0.00(0.0-1.0)$ & $0.00(0.0-0.0)$ & 0.002 & 0.022 \\
\hline Resting asymmetry score (0-20) & $5.00(0.0-15.0)$ & $0.00(0.0-0.0)$ & $<0.001$ & $10.00(0.0-15.0)$ & $0.00(0.0-5.0)$ & $<0.001$ & 0.686 \\
\hline Forehead wrinkle (1-5) & $2.00(1.0-3.8)$ & $5.00(5.0-5.0)$ & $<0.001$ & $2.00(1.0-2.0)$ & $5.00(5.0-5.0)$ & $<0.001$ & 0.399 \\
\hline Gentle eye closure (1-5) & $2.00(1.0-3.0)$ & $5.00(5.0-5.0)$ & $<0.001$ & $2.00(1.0-2.0)$ & $5.00(4.0-5.0)$ & $<0.001$ & 0.553 \\
\hline Open mouth smile (1-5) & $2.00(1.0-4.0)$ & $5.00(5.0-5.0)$ & $<0.001$ & $2.00(1.0-2.0)$ & $5.00(4.0-5.0)$ & $<0.001$ & 0.331 \\
\hline Snarl (1-5) & $2.00(1.0-3.0)$ & $5.00(5.0-5.0)$ & $<0.001$ & $2.00(1.0-2.0)$ & $5.00(4.0-5.0)$ & $<0.001$ & 0.587 \\
\hline Lip pucker (1-5) & $2.00(1.0-3.0)$ & $5.00(5.0-5.0)$ & $<0.001$ & $2.00(1.0-2.0)$ & $5.00(4.0-5.0)$ & $<0.001$ & 0.498 \\
\hline Symmetry voluntary movement (20-100) & $40.00(20.0-68.0)$ & $100.00(92.0-100)$ & $<0.001$ & $36.00(20.0-60.0)$ & $100.00(84.0-100)$ & $<0.001$ & 0.528 \\
\hline
\end{tabular}

*Median and interquartile range differences within groups.

+ Median and interquartile range differences between groups.

C+FNT: corticosteroids plus facial neuromuscular training; FNT: facial neuromuscular training; SB-FGS: Sunnybrook facial grading system; HB-FGS: House-Brackmann facial grading system.

Table 4. the $\Delta$ of HB-FGS and $\Delta$ of SB-FGS, after intervention-baseline intra-groups and between groups.

\begin{tabular}{|c|c|c|c|}
\hline Scales & $\mathrm{C}+\mathrm{FNT}$ & FNT & $p$-value + \\
\hline HB-FGS (I-VI grade) & $-2.00(-3.0$. to -2.0$)$ & $-2.00(-3.0$ to -2.0$)$ & 0.996 \\
\hline Composite facial symmetry score $(0-100)$ & 54.50 (28.0 to 77.3$)$ & 47.00 (16.0 to 76.0$)$ & 0.476 \\
\hline Eye $(0-1)$ & $-1.00(-1.0$ to 0.0$)$ & $0.00(-1.0$ to 0.0$)$ & 0.889 \\
\hline Cheek (0-2) & $0.00(-1.0$ to 0.0$)$ & $-1.00(-1.0$ to 0.0$)$ & 0.761 \\
\hline Mouth $(0-1)$ & $0.00(-1.0$ to 0.0$)$ & $0.00(-1.0$ to 0.0$)$ & 0.921 \\
\hline Resting asymmetry score $(0-20)$ & $-5.00(-15$ to 0.0$)$ & $-5.00(-15.0$ to 0.0$)$ & 0.648 \\
\hline Open mouth smile $(1-5)$ & $2.00(1.0$ to 3.0$)$ & $2.00(1.0$ to 3.0$)$ & 0.631 \\
\hline Snarl (1-5) & $3.00(1.0$ to 3.0$)$ & $2.00(1.0$ to 3.0$)$ & 0.575 \\
\hline Lip pucker (1-5) & $2.00(1.3$ to 3.0$)$ & $2.00(1.0$ to 3.0$)$ & 0.532 \\
\hline Symmetry voluntary movement $(20-100)$ & 50.00 (28.0 to 66.0$)$ & $44.00(16.0$ to 64.0$)$ & 0.487 \\
\hline
\end{tabular}

+ Change after intervention-baseline between groups.

C + FNT: corticosteroids plus facial neuromuscular training; FNT: facial neuromuscular retraining; HB-FGS: House-Brackmann facial grading system; SB-FGS: Sunnybrook facial grading system; $\Delta$ : final values-initial values.

Table 5. Patients' distribution among HB-FGS grades at the beginning and after 6 weeks of intervention.

\begin{tabular}{|c|c|c|c|c|c|c|c|}
\hline \multirow[b]{2}{*}{ HB-FGS } & \multicolumn{2}{|c|}{$\mathrm{C}+\mathrm{FNT}(n=42)$} & \multirow[b]{2}{*}{$p$-value* } & \multicolumn{2}{|c|}{ FNT $(n=31)$} & \multirow[b]{2}{*}{$p$-value* } & \multirow[b]{2}{*}{$p$-value +} \\
\hline & Baseline & After 6 weeks & & Baseline & After 6 weeks & & \\
\hline HB grade I & $0(0 \%)$ & 27 (64.29\%) & $<0.001$ & $0(0 \%)$ & $20(64.52 \%)$ & $<0.001$ & 0.992 \\
\hline HB grade II-IV & $22(52.38 \%)$ & $13(30.95 \%)$ & $<0.001$ & $16(51.61 \%)$ & 7 (22.58\%) & $<0.001$ & 0.777 \\
\hline HB grade V/VI & $20(47.62 \%)$ & $2(4.76 \%)$ & $<0.001$ & 15 (48.39\%) & $4(12.90 \%)$ & $<0.001$ & 0.703 \\
\hline
\end{tabular}

C+FNT: corticosteroids plus facial neuromuscular training; FNT: facial neuromuscular training; HB-FGS: House-Brackmann facial grading system.

*intra-group $p \leq 0.001$.

+ between groups.

Table 6. Relationship between the independent and predictive variables.

\begin{tabular}{lcccrrr}
\hline & \multicolumn{2}{c}{ Unstandardized coefficients } & & \multicolumn{2}{c}{$\begin{array}{c}\text { Standardized } \\
\text { coefficients }\end{array}$} \\
\cline { 2 - 3 } Independent variables & B $(95 \% \mathrm{Cl})$ & SE & & $B$ & $p$-value \\
\hline Constant & $2.615(1.352-3.878)$ & 0.633 & & $<0.001$ \\
Intervention groups & $0.024(-0.440-0.488)$ & 0.233 & & 0.010 & 0.917 \\
Age & $-0.018(-0.030-0.006)$ & 0.006 & & -0.285 & 0.004 \\
Sex (male) & $-0.705(-1.154-0.255)$ & 0.225 & & -0.292 & 0.003 \\
Baseline of HB-FGS & $0.393(0.241-0.545)$ & & 0.076 & & 0.480 & $<0.001$ \\
\hline
\end{tabular}

B: Unstandardized Coefficient; $B$ : Standardized Coefficient; SE: Standard Error; Independent variables: age, sex, baseline HB-FGS, and intervention groups; Predictor: $\Delta$ of recovery degree (HB-FGS).

not prescribed to all patients with the same severity level of BP.

This study showed that, after 6 weeks, both interventions reduced the degree of BP severity in a similar way. At the pre-intervention phase, both groups had similar distribution of facial grades' severity, which supports the concept that post-treatment values provided a reliable evaluation of each treatment efficacy. After interventions there were no significant differences between groups for all components of facial symmetry at rest and voluntary movement, except for two parameters (resting symmetry component of cheek and mouth), revealing that corticosteroids only had positive effects on rest symmetry with droop corner of mouth and increased nasal labial fold. Our results do not support the present treatment guidelines, which advocate the use of potent anti-inflammatory agents, such as oral corticosteroids, for all patients with BP to decrease recovery time and improve facial nerve functional recovery $[4,9,10]$. These guidelines were based on the assumption that an early administration of corticosteroids might reduce the nerve's swelling and subsequent unblocking of nerve conduction, in the fallopian canal [1]. These results may suggest that the corticosteroids dose was insufficient to promote anti-inflammatory effects in the fallopian canal. The oral administration has a systemic effect, which may have 


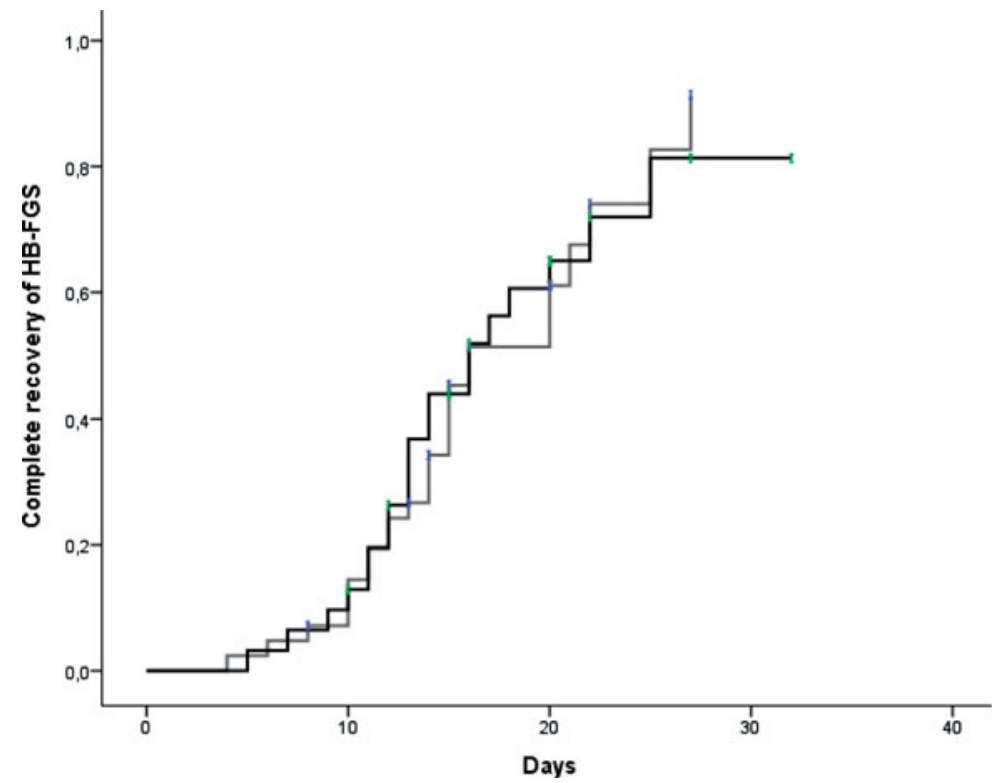

\begin{tabular}{lcccc} 
Incomplete recovery & $\mathbf{d 0}$ & $\mathbf{d} \leq \mathbf{1 0}$ & $\mathbf{d} \leq \mathbf{2 0}$ & $\mathbf{d} \leq \mathbf{3 0}$ \\
\hline C+FNT group & $42(100 \%)$ & $3(85.7 \%)$ & $19(45.2 \%)$ & $15(35.7 \%)$ \\
\hline FNT group & $31(100 \%)$ & $27(87.1 \%)$ & $14(41.9 \%)$ & $11(35.5 \%)$ \\
\hline
\end{tabular}

$\mathrm{C}+\mathrm{FNT}$, corticosteroids plus facial neuromuscular retraining; FNT, facial neuromuscular training; HB-FGS, House-Brackmann facial grading scale

Figure 1. Hazard curves for complete recovery, according to intervention group.

compromised the local effect at the fallopian canal. Independently of the underlying reasons, our results suggest that the criteria to prescribe corticosteroids in BP must be revised. Other variables may influence the impact of corticosteroids, including the specific pharmacologic agent (methylprednisolone, prednisolone, deflazacort, or dexamethasone) and duration (short vs long duration).

The homogeneous distribution of BP severity among the groups at the beginning of the interventions clearly suggests the absence of standard criteria to prescribe corticosteroids by clinical physicians, once there are slight-to-moderate degrees (HB-FGS/grade II-IV) and severe degrees (HB-FGS/ grade $\mathrm{V}-\mathrm{VI}$ ) of $\mathrm{BP}$ with and without corticosteroids therapy. Fortunately, this absence of uniform criteria allows us to obtain a great homogeneity in the distribution of BP severity between groups at the beginning of the experimental protocol, demonstrating also that both interventions had similar effects in all recovery degrees of patients, independently of severity level at baseline $(p=0.992)$. Unlike our results, another study showed that drugs therapy (corticosteroids and valacyclovir) plus facial exercises had significant effect on the grade $(p=0.038)$ and time $(p=0.044)$ of full recovery in patients with severe facial palsy (HB-FGS/grade V-VI) [15]. However, differences in the characteristics of the enrolled patients (HB grade IV and V-VI vs II-IV and V/VI), intervention types and biased variables' control (drugs therapy+FNT and drugs therapy vs $\mathrm{C}+\mathrm{FNT}$ and FNT only), and length of follow-up (6 months vs 6 weeks) limit a direct comparison between studies.

Both interventions showed positive results (HB-FGS and SB-FGS), but the occurrence of spontaneous recovery in the acute phases of BP may have influenced our results. In fact, an early study demonstrated that spontaneous recovery of the facial nerve follows a predictable time course after an injury, reporting a complete recovery of $85 \%$ of patients within 3 weeks [1]. Peitersen [1] showed that full spontaneous recovery may not occur in all patients with facial nerve paralysis and, for this reason, various therapies $[5,11,15]$ have been designed to enhance functional recovery and reduce sequels of BP. Facial exercise is a conservative intervention for this condition and is considered to be an effective form of treatment $[6,11,15]$, although its effects have been found to be debatable according to different guidelines $[9,10]$. FNT provides specific exercises and strategies based on individual function, muscle characteristics, and degree of facial neuromuscular damage (symptoms and clinical signs) [11]. This approach integrates the fields of therapeutic, behavioral, and educational science with the goal of restoring muscular function, and prevents irregular muscular activity, as much as possible, in order to improve facial functions (eating, drinking, speaking clearly, and expressing emotions) [11]. Although there is no compelling evidence about the effectiveness of FNT intervention, some studies have recognized the efficacy of functional recovery on early stages of BP [16,17]. Theoretically, manual mechanism stimulation directly affects the enervated muscles fibers by increasing the circulation, maintaining membrane properties, and consequently improving the motor response [18].

Finally, our results showed that age, sex, and level of baseline HB-FGS might be useful to predict the recovery degree, irrespective of the intervention groups. Similarly to our results, other studies have also reported that old patients 
have a poor prognosis, which may be due to an age-related increase in brain cytokine activity that impairs the ability of cell repair [19]. Our results showed a better prognosis for females, which might be explained, among others factors, by the influence of the endocrine environment, once the antiinflammatory role with favorable effects of estrogens in many diseases are well known [20]. Further research is necessary to clarify the potential effect of gender as a predictor factor to full recovery of $\mathrm{BP}$.

\section{Study limitations}

This study had both strengths and limitations. The strengths of the study included the rigorous control to reduce bias through blinded assessment and patient's selection (homogenous population with exclusion of many comorbidity). The limitations of the study were the lack of a control group to assess spontaneous recovery, which restricts the analysis of FNT effectiveness. However, a control group was not included due to ethical reasons.

\section{Conclusions}

Our results provide preliminary evidence that the oral intake of corticosteroids was not effective in all grades of dysfunction and remission time at the early phase of BP, highlighting the need to re-analyze the criteria to prescribe corticosteroids in these patients.

\section{Disclosure statement}

The authors report no conflicts of interest. The authors alone are responsible for the content and writing of the paper.

\section{References}

[1] Peitersen E. Bell's palsy: the spontaneous course of 2,500 peripheral facial nerve palsies of different etiologies. Acta Otolaryngol 2002;549:4-30.

[2] Ishii L, Godoy A, Encarnacion CO, Byrne PJ, Boahene KD, Ishii M. Not just another face in the crowd: society's perceptions of facial paralysis. Laryngoscope 2012;122:533-8.

[3] Dawidowsky K, Branica S, Batelja L, Dawidowsky B, Kovac-Bilic L, Simunic-Veselic A. Anatomical study of the facial nerve canal in comparison to the site of the lesion in bell's palsy. Coll Antropol 2011;35:61-5.
[4] Gronseth GS, Paduga R. Evidence-based guideline update: steroids and antivirals for Bell palsy: report of the Guideline development subcommittee of the American academy of neurology. Neurology 2012;79:2209-13.

[5] Sullivan FM, Swan IR, Donnan PT, et al. Early treatment with prednisolone or acyclovir in Bell's palsy. $\mathrm{N}$ Engl J Med 2007;357:1598-607.

[6] Pereira LM, Obara K, Dias JM, Menacho MO, Lavado EL, Cardoso JR. Facial exercise therapy for facial palsy: systematic review and meta-analysis. Clin Rehabil 2010;25:649-58.

[7] Teixeira LJ, Soares BGDO, Vieira VP, Prado GF. Physical therapy for Bell's palsy (idiopathic facial paralysis). Cochrane Database Syst Rev 2011;3:CD006283.

[8] Ferreira M, Santos PC, Duarte J. Idiopathic facial palsy and physical therapy: an intervention proposal following a review of practice-systematic review. Phys Ther Rev 2011;16:237-43.

[9] Almeida JR, Guyatt GH, Sud S, Dorion J, Hill MD, Kolber MR, et al. Management of Bell palsy: clinical practice guideline. Cmaj 2014;186:917-22.

[10] Murthy JMK, Saxena AB. Bell's palsy: treatment guidelines. Ann Indian Acad Neurol 2011;14:S70-2.

[11] VanSwearingen J. Facial rehabilitation: A neuromuscular reeducation, patient-centered approach. Facial Plast Surg 2008;24: 250-9.

[12] Garber CE, Blissmer B, Deschenes MR, Franklin BA, Lamonte MJ, Lee IM, et al. American College of Sports Medicine. American College of Sports Medicine position stand. Quantity and quality of exercise for developing and maintaining cardiorespiratory, musculoskeletal, and neuromotor fitness in apparently healthy adults: guidance for prescribing exercise. Med Sci Sports Exerc 2011;43:1334-59.

[13] Evans RA, Harries ML, Baguley DM, Moffat DA. Reliability of the house and Brackmann grading system for facial palsy. J Laryngol Otol 1989;103:1045-6.

[14] Ross BR, Fradet G, Nedzelski JM. Development of a sensitive clinical facial grading system. Otolaryngol Head Neck Surg 1996;114:380-6.

[15] Nicastri M, Mancini P, De Seta D, et al. Efficacy of early physical therapy in severe Bell's palsy: a randomized controlled trial. Neurorehabil Neural Repair 2013;17:542-51.

[16] Manikandan N. Effect of facial neuromuscular re-education on facial symmetry in patients with Bell's palsy: a randomized controlled trial. Clin Rehabil 2007;21:338-43.

[17] Barbara M, Antonini G, Vestri A, Volpini L, Monini S. Role of Kabat physical rehabilitation in Bell's palsy: a randomized trial. Acta Otolaryngol 2010;130:167-72.

[18] Diels HJ. Facial paralysis: is there a role for a therapist? Facial Plast Surg 2000;16:361-4.

[19] Hsieh R-L, Wu C-H, Wang L-I, Lee W-C. Correlates of degree of nerve involvement in early Bell's palsy. BMC Neurol 2009;9:1-5.

[20] Straub RH. The Complex Role of Estrogens in Inflammation. Endocrine Rev 2007;28:521-74. 